\title{
ON THE H-SYSTEMS IN HIGHER DIMENSION
}

\author{
NICOLA FUSCO, JAN KRISTENSEN, CHIARA LEONE AND ANNA VERDE
}

AbSTRACT. We prove a regularity result for the weak solutions of H-systems in dimensions $n \geq 3$.

2010 Mathematics Subject Classification: Primary 35J47; Secondary 35J25

Key words: Elliptic system, regularity of solutions

\section{INTRODUCTION}

Let $\Omega$ be a bounded Lipschitz domain in $\mathbb{R}^{n}$. For a given bounded Borel function $H: \mathbb{R}^{n+1} \rightarrow \mathbb{R}$ the corresponding $H$-system on $\Omega$ is the elliptic system

$$
-\operatorname{div}\left(|\nabla u|^{n-2} \nabla u\right)=n^{\frac{n}{2}} H(u) J_{u}
$$

where $u: \Omega \rightarrow \mathbb{R}^{n+1}$ is of Sobolev class $\mathrm{W}^{1, n}$ and $J_{u}=u_{x_{1}} \wedge \cdots \wedge u_{x_{n}}$ is the cross product of the partial derivatives $u_{x_{j}}$ of $u$. Recall that the cross product $v_{1} \wedge \cdots \wedge v_{n}$ of $n$ vectors $v_{1}, \ldots, v_{n}$ in $\mathbb{R}^{n+1}$ is defined as the unique vector in $\mathbb{R}^{n+1}$ satisfying for all $w \in \mathbb{R}^{n+1}$,

$$
w \cdot\left(v_{1} \wedge \cdots \wedge v_{n}\right)=\operatorname{det}\left[\begin{array}{ccc}
w^{1} & \cdots & w^{n+1} \\
v_{1}^{1} & \cdots & v_{1}^{n+1} \\
\vdots & & \vdots \\
v_{n}^{1} & \cdots & v_{n}^{n+1}
\end{array}\right] .
$$

where we write $v_{j}=\left(v_{j}^{1}, \ldots, v_{j}^{n+1}\right), w=\left(w^{1} \ldots, w^{n+1}\right)$.

Definition 1.1. A map $u \in \mathrm{W}^{1, n}\left(\Omega, \mathbb{R}^{n+1}\right)$ is a weak solution to (1.1) if, for any test map $\varphi \in$ $\mathrm{C}_{c}^{\infty}\left(\Omega, \mathbb{R}^{n+1}\right)$

$$
\int_{\Omega}|\nabla u|^{n-2} \nabla u \cdot \nabla \varphi \mathrm{d} x=n^{\frac{n}{2}} \int_{\Omega} H(u) \varphi \cdot J_{u} \mathrm{~d} x
$$

holds.

Remark 1.2. By approximation it can be easily seen that equation (1.2) can be tested by any map $\varphi \in \mathrm{W}_{0}^{1, n}\left(\Omega, \mathbb{R}^{n+1}\right) \cap \mathrm{L}^{\infty}\left(\Omega, \mathbb{R}^{n+1}\right)$.

It is well-known that if, in addition to (1.1), $u$ is $\mathrm{C}^{2}$ and conformal meaning that for some nonnegative function $\lambda$,

$$
u_{x_{i}} \cdot u_{x_{j}}=\lambda \delta_{i j}
$$

holds on $\Omega$ for all $i, j$, then the image $u(\Omega)$ is a surface whose mean curvature is $H(u(x))$ at each point $u(x)$ where $J_{u}(x) \neq 0$. For $n=2$ this observation is the starting point for most existence results for parametric surfaces of prescribed mean curvature, see $[4,25]$. The conformality condition will play no direct role in the present paper.

The $H$-system (1.1) is the Euler-Lagrange system for the variational integral

$$
\mathcal{E}(u)=\int_{\Omega}\left(\frac{1}{n}|\nabla u|^{n}+n^{\frac{n}{2}} Q_{H}(u) \cdot J_{u}\right) \mathrm{d} x
$$

where $Q_{H}: \mathbb{R}^{n+1} \rightarrow \mathbb{R}^{n+1}$ is any vector field such that $\operatorname{div} Q_{H}=H$ on $\mathbb{R}^{n+1}$. This has been used to prove existence of solutions by either constrained minimization (small solutions) or methods based on Mountain Pass type theorems (large solutions), see for instance [6, 10, 20] and [4], [25, Ch. III.5] for a 
more comprehensive discussion. Existence has also recently been deduced by use of a heat flow for the $H$-system [18].

Here we shall focus entirely on the regularity of weak solutions to the $H$-system (1.1) in dimension $n \geq 3$ under additional assumptions on the function $H$ or the weak solution $u$. The regularity of (1.1) in the two dimensional case $n=2$ has been well-studied and culminated in Rivière's solution of the Heinz conjecture in [21]: under the sole assumption of boundedness of $H$, the weak solution defined in Definition 1.1 is continuous. Many authors contributed with important results prior to this, including $[16,26,28,3,15,2]$. The higher dimensional case $n \geq 3$ seems to be more difficult and the results are far from being conclusive. We refer to [22] for a more comprehensive discussion of the background literature. In this connection remark that (1.1) is a nonlinear degenerate elliptic system with critical growth nonlinearity $H(u) J_{u}$ on the right-hand side that is merely integrable for maps $u$ of class $\mathrm{W}^{1, n}$. It is well-known that such systems in general admit very singular solutions and that a general regularity theory is only possible provided the right-hand side nonlinearity has a special structure (see for instance [1]). As mentioned, this is fully exploited for the system in two dimensions $n=2$ by Rivière's result, and the hope is that the structure of $H(u) J_{u}$ remains strong enough to ensure regularity also for $n \geq 3$, at least when backed up by appropriate additional hypotheses on $H$. We mention the results of Mou and Yang [20] who proved that solutions of (1.1) are $\mathrm{C}^{1}$, provided that either $H$ is constant or $u$ is weakly conformal. Closer to our result is that of Wang [27] who proved a higher-dimensional generalization of Heinz's result [16] under the assumption

$$
\sup _{y \in \mathbb{R}^{n+1}}\left(|H(y)|+(1+|y|)\left|H^{\prime}(y)\right|\right)<\infty .
$$

His proof is based on the the coarea formula and thus follows to some extent the proof of Bethuel [2] for the two dimensional case. Here we are able to prove the following result:

Theorem 1.3. Let $H: \mathbb{R}^{n+1} \rightarrow \mathbb{R}$ be a locally Lipschitz function satisfying the two conditions:

$$
y \mapsto H(y) y \text { is uniformly continuous on } \mathbb{R}^{n+1}
$$

and for some exponent $q>1$,

$$
\sup _{y \in \mathbb{R}^{n+1}}\left(|H(y)|+\frac{\left|H^{\prime}(y)\right|}{1+|y|^{q}}\right)<\infty .
$$

Then any weak solution in the sense of Definition 1.1 is of class $\mathrm{C}_{\mathrm{loc}}^{1, \alpha}$ on $\Omega$ for some $\alpha \in(0,1)$.

We refer to Section 4 for examples of functions $H$ that fail to satisfy the Heinz-Wang condition (1.3) but do satisfy our conditions (1.4)-(1.5). The conditions (1.4)-(1.5) serve mainly to ensure that the weak solutions are locally bounded. Indeed if we assume a priori that the weak solution is locally bounded, then much weaker conditions on $H$ suffice to conclude higher regularity:

Proposition 1.1. Let $H: \mathbb{R}^{n+1} \rightarrow \mathbb{R}$ be continuous. Then any locally bounded weak solution to (1.1) is locally Hölder continuous. Furthermore, if $H$ is locally Lipschitz, then any locally bounded weak solution to (1.1) is of class $\mathrm{C}_{\mathrm{loc}}^{1, \alpha}$ on $\Omega$ for some $\alpha \in(0,1)$.

The proofs of Theorem 1.3 and Proposition 1.1 are given in Section 3.

Acknowledgement. N. Fusco, C. Leone and A. Verde are members of the Gruppo Nazionale per l'Analisi Matematica, la Probabilità e le loro Applicazioni (GNAMPA) of the Istituto Nazionale di Alta Matematica (INdAM)

\section{PRELIMINARY RESUlTS}

We start this section by recalling some notions from harmonic analysis that allow us to take advantage of the Jacobian structure on the right-hand side of (1.1). In the process of doing that we also fix our notation. As regards function spaces and Sobolev functions it is standard and follows [19, 29]. On 
$\mathbb{R}^{n}, \mathbb{R}^{n+1}$ and matrix space $\mathbb{R}^{(n+1) \times n}$ we use standard euclidean inner products and the corresponding norms. In all cases denoted by $X \cdot Y$ and $|X|=\sqrt{X \cdot X}$, respectively, the meaning being clear from the context.

Recall that a function $f \in \mathrm{L}^{1}\left(\mathbb{R}^{n}\right)$ belongs to the Hardy space $\mathcal{H}^{1}\left(\mathbb{R}^{n}\right)$ if

$$
f_{*}:=\sup _{\varepsilon>0}\left|\phi_{\varepsilon} * f\right| \in \mathrm{L}^{1}\left(\mathbb{R}^{n}\right),
$$

where as usual we let $\phi_{\varepsilon}(x):=\varepsilon^{-n} \phi(x / \varepsilon)$ for a fixed nonnegative function $\phi \in \mathrm{C}_{c}^{\infty}\left(B_{1}(0)\right)$ with $\int \phi(y) d y=1$. The definition does not depend on the choice of $\phi$ and the expression $\|f\|_{\mathcal{H}^{1}}:=\left\|f_{*}\right\|_{1}$ defines a norm in $\mathcal{H}^{1}\left(\mathbb{R}^{n}\right)$. Hereby $\mathcal{H}^{1}\left(\mathbb{R}^{n}\right)$ is a Banach space whose dual can be identified with the John-Nirenberg space BMO of functions of bounded mean oscillation. To define $\mathrm{BMO}\left(\mathbb{R}^{n}\right)$ denote for $g \in \mathrm{L}_{\text {loc }}^{1}\left(\mathbb{R}^{n}\right)$ and a ball $B$ in $\mathbb{R}^{n}$ the integral mean of $g$ on $B$ by

$$
g_{B}:=\frac{1}{\mathscr{L}^{n}(B)} \int_{B} g \mathrm{~d} x:=f_{B} g \mathrm{~d} x .
$$

Now $g \in \operatorname{BMO}\left(\mathbb{R}^{n}\right)$ provided $g \in \mathrm{L}_{\text {loc }}^{1}\left(\mathbb{R}^{n}\right)$ and

$$
\|g\|_{\mathrm{BMO}}:=\sup _{B} f_{B}\left|g-g_{B}\right| \mathrm{d} x
$$

is finite, where the supremum is taken over all balls $B$ in $\mathbb{R}^{n}$. The importance of BMO here is due to the elementary fact that any Sobolev function of class $\mathrm{W}^{1, n}\left(\mathbb{R}^{n}\right)$ in particular is BMO: $\|g\|_{\mathrm{BMO}} \leq$ $c\|\nabla g\|_{\mathrm{L}^{n}}$ for a dimensional constant $c=c(n)$ by Poincaré's inequality.

On the other hand, Coifman, Lions, Meyer and Semmes in [9] established the crucial connection between the Jacobian determinant of a map in $\mathrm{W}^{1, n}\left(\mathbb{R}^{n}, \mathbb{R}^{n}\right)$ and the Hardy space $\mathcal{H}^{1}\left(\mathbb{R}^{n}\right)$ that we also record here for later reference:

Theorem 2.1. If $f \in \mathrm{W}^{1, n}\left(\mathbb{R}^{n}, \mathbb{R}^{n}\right)$, then $\operatorname{det} \nabla f \in \mathcal{H}^{1}\left(\mathbb{R}^{n}\right)$ and

$$
\|\operatorname{det} \nabla f\|_{\mathcal{H}^{1}} \leq C\|\nabla f\|_{\mathrm{L}^{n}}^{n}
$$

where $C=C(n)$.

Finally, as already mentioned BMO can be identified with the dual space of $\mathcal{H}^{1}$, and this is conveniently expressed through Fefferman's duality inequality [12]:

$$
\int_{\mathbb{R}^{n}} f g \mathrm{~d} x \leq c\|f\|_{\mathcal{H}^{1}}\|g\|_{\text {BMO }}
$$

valid for all $f \in \mathcal{H}^{1}, g \in$ BMO. In general, the integral on the left-hand side of (2.2) does not converge, but there are a number of ways to give meaning to it [23].

Having disposed of these harmonic analysis tools we turn to the uniform continuity assumption (1.4) in Theorem 1.3. Define the truncation at level $k>0$ by $T_{k}(u):=u \psi_{k}(|u|)$, where

$$
\psi_{k}(t):= \begin{cases}1 & \text { if } t \leq k \\ \frac{k}{t} & \text { if } t>k .\end{cases}
$$

Lemma 2.2. If $y \mapsto H(y) y$ is uniformly continuous and $H$ is bounded, then there exists an increasing concave modulus of continuity $\omega:[0, \infty) \rightarrow[0, \infty)$ such that

$$
\left|H\left(y_{1}\right) T_{k}\left(y_{1}\right)-H\left(y_{2}\right) T_{k}\left(y_{2}\right)\right| \leq \omega\left(\left|y_{1}-y_{2}\right|\right)
$$

holds for all $y_{1}, y_{2} \in \mathbb{R}^{n+1}$ and all $k>0$.

Proof. Let $\theta$ denote a modulus of continuity for $y \mapsto H(y) y$. We can assume that $\theta$ is concave and increasing. We get for $y_{1}, y_{2} \in \mathbb{R}^{n+1},\left|y_{2}\right| \leq\left|y_{1}\right|, k>0$ :

$$
\begin{aligned}
\left|H\left(y_{1}\right) T_{k}\left(y_{1}\right)-H\left(y_{2}\right) T_{k}\left(y_{2}\right)\right| \leq & \left|H\left(y_{1}\right) y_{1}-H\left(y_{2}\right) y_{2}\right| \psi_{k}\left(\left|y_{1}\right|\right) \\
& +\left|H\left(\left|y_{2}\right|\right) y_{2}\right|\left|\psi_{k}\left(\left|y_{1}\right|\right)-\psi_{k}\left(\left|y_{2}\right|\right)\right| \\
\leq & \theta\left(\left|y_{1}-y_{2}\right|\right)+C\left|y_{2}\right|\left|\psi_{k}\left(\left|y_{1}\right|\right)-\psi_{k}\left(\left|y_{2}\right|\right)\right| .
\end{aligned}
$$


If $\left|y_{1}\right|,\left|y_{2}\right| \leq k$ we get $\left|\psi_{k}\left(\left|y_{1}\right|\right)-\psi_{k}\left(\left|y_{2}\right|\right)\right|=0$. If $\left|y_{1}\right|,\left|y_{2}\right|>k$, then the last term becomes

$$
C\left|y_{2}\right|\left|\frac{k}{\left|y_{1}\right|}-\frac{k}{\left|y_{2}\right|}\right|=C\left|y_{2}\right| k \frac{|| y_{1}|-| y_{2}||}{\left|y_{1}\right|\left|y_{2}\right|} \leq c\left|y_{1}-y_{2}\right| .
$$

If $\left|y_{1}\right|>k$ and $\left|y_{2}\right| \leq k$, then the last term can be estimated as

$$
C\left|y_{2}\right|\left|\frac{k}{\left|y_{1}\right|}-1\right|=C \frac{\left|y_{2}\right|}{\left|y_{1}\right|}|k-| y_{1}|| \leq C\left[\left|y_{1}\right|-k\right] \leq C|| y_{1}|-| y_{2}|| \leq C\left|y_{1}-y_{2}\right| .
$$

Thus we have shown that we may take $\omega(t)=\theta(t)+C|t|$, concluding the proof.

Lemma 2.3. For $u \in \mathrm{W}_{\text {loc }}^{1, n}\left(\Omega, \mathbb{R}^{n+1}\right)$ we have for any ball $B \Subset \Omega$ and any $k>0$ that

$$
f_{B}\left|T_{k}(u) H(u)-\left(T_{k}(u) H(u)\right)_{B}\right| \mathrm{d} x \leq c \omega\left(\left(\int_{B}|\nabla u|^{n} \mathrm{~d} x\right)^{\frac{1}{n}}\right),
$$

where $c=c(n)$ is a constant, $\omega$ is determined in Lemma 2.2 and $|\cdot|$ denotes standard euclidean norm (in $\mathbb{R}^{n+1}$ and in $\mathbb{R}^{(n+1) \times n}$, respectively).

Proof. We simply estimate:

$$
\begin{array}{cll}
f_{B}\left|T_{k}(u) H(u)-\left(T_{k}(u) H(u)\right)_{B}\right| d x & \leq & 2 f_{B}\left|T_{k}(u) H(u)-T_{k}\left(u_{B}\right) H\left(u_{B}\right)\right| d x \\
& \stackrel{\text { Lemma } 2.2}{\leq} & 2 f_{B} \omega\left(\left|u-(u)_{B}\right|\right) d x \\
& \stackrel{\text { Jensen }}{\leq} & 2 \omega\left(f_{B}\left|u-(u)_{B}\right| d x\right) \\
& \stackrel{\text { Poincare-Wirtinger }}{\leq} & 2 \omega\left(C\left(\int_{B}|\nabla u|^{n} d x\right)^{\frac{1}{n}}\right) .
\end{array}
$$

Finally since $C \geq 1$ (without loss of generality) and $\omega$ is concave we have for each $t>0$ that $\omega(C t) / C t \leq \omega(t) / t$ and therefore $\omega(C t) \leq C \omega(t)$, thus concluding the proof.

The next result is well-known, but as we require it in a special form we prefer to state and derive it explicitly:

Lemma 2.4. There exists a dimensional constant $C_{n}$ with the following property. For each $u \in$ $W^{1, n}\left(B_{r}\left(x_{0}\right)\right)$ there exists $\bar{u} \in \mathrm{W}_{\mathrm{loc}}^{1, n}\left(\mathbb{R}^{n}\right)$ such that $\bar{u}=u$ on $B_{r}\left(x_{0}\right)$ and

$$
\int_{\mathbb{R}^{n}}|\nabla \bar{u}|^{n} \mathrm{~d} x \leq C_{n} \int_{B_{r}\left(x_{0}\right)}|\nabla u|^{n} \mathrm{~d} x .
$$

Furthermore we can arrange that

$$
\sup _{x \in \mathbb{R}^{n} \backslash B_{r}\left(x_{0}\right)}|\bar{u}(x)| \leq\left|u_{B_{r}\left(x_{0}\right)}\right|+\sup _{x \in \partial B_{r}\left(x_{0}\right)}|u(x)|
$$

where the right-hand side could be infinite (making the bound vacuous).

Proof. Considering the function $x \mapsto u\left(x_{0}+r x\right) / r$ instead of $u$ we may assume that $B_{r}\left(x_{0}\right)=$ $B_{1}(0)=: B$, the open unit ball in $\mathbb{R}^{n}$. For Lipschitz $v: \bar{B} \rightarrow \mathbb{R}$ we put $m=\sup _{\partial B}|v|$ and

$$
V(x)= \begin{cases}v(x) & \text { if } x \in B \\ T_{m}\left(v\left(\frac{x}{|x|^{2}}\right)(2-|x|)^{+}\right) & \text {if } x \in \mathbb{R}^{n} \backslash B,\end{cases}
$$

where $T_{m}: \mathbb{R} \rightarrow \mathbb{R}$ is the 1-dimensional version of the truncation map defined above (so $T_{m}(t):=$ $\max \{\min \{t, m\},-m\})$. Then $V: \mathbb{R}^{n} \rightarrow \mathbb{R}$ is Lipschitz, $V=v$ on $B, V=0$ on $\mathbb{R}^{n} \backslash 2 B$ and $|V| \leq m$ on $\mathbb{R}^{n} \backslash B$. For $1<|x|<2$ with $\left|v\left(\frac{x}{|x|^{2}}\right)\right|(2-|x|)<m$ we have almost everywhere

$$
\nabla V(x)=(2-|x|) \nabla v\left(\frac{x}{|x|^{2}}\right) \frac{I-2 \frac{x \otimes x}{|x|^{2}}}{|x|^{2}}-v\left(\frac{x}{|x|^{2}}\right) \frac{x}{|x|},
$$


while if $\left|v\left(\frac{x}{|x|^{2}}\right)\right|(2-|x|) \geq m$ we have $\nabla V(x)=0$ almost everywhere. Thus after a routine estimation, involving also a change of variables, we find

$$
\int_{\mathbb{R}^{n}}|\nabla V|^{n} \mathrm{~d} x \leq c \int_{B}\left(|\nabla v|^{n}+|v|^{n}\right) \mathrm{d} x
$$

for some dimensional constant $c=c(n)$. It follows by approximation that the above construction and listed properties extend by continuity to functions $v \in \mathrm{W}^{1, n}(B)$ : there exists $V \in \mathrm{W}^{1, n}\left(\mathbb{R}^{n}\right)$ so $\left.V\right|_{B}=$ $v,\left.V\right|_{\mathbb{R}^{n} \backslash 2 B}=0, \sup _{\mathbb{R}^{n} \backslash B}|V| \leq \sup _{\partial B}|v|$ and (2.6) hold. To complete the proof let $u \in \mathrm{W}^{1, n}(B)$ and put $v=u-u_{B}$, where $u_{B}$ is the integral mean of $u$ over $B$. Then $\bar{u}=V+u_{B}$ belongs to $\mathrm{W}_{\text {loc }}^{1, n}\left(\mathbb{R}^{n}\right)$ and the desired bounds follow by combination of the above and Poincaré's inequality.

A useful feature of the determinant expression $J_{u}$ on the right-hand side of (1.1) involves also the truncation maps $T_{k}$ defined above and is summarized in the next

Lemma 2.5. For $\varphi \in \mathrm{C}_{c}^{1}(\Omega), u \in \mathrm{W}_{\text {loc }}^{1, n}\left(\Omega, \mathbb{R}^{n+1}\right)$ and $k>0$

$$
\varphi^{n} T_{k}(u) \cdot J_{u}=T_{k}(u) \cdot J_{\varphi u} .
$$

Proof. By definition the right-hand side equals

$$
T_{k}(u) \cdot J_{\varphi u}=\left[\begin{array}{ccc}
T_{k}(u)^{1} & \cdots & T_{k}(u)^{n+1} \\
\varphi_{x_{1}} u^{1}+\varphi u_{x_{1}}^{1} & \cdots & \varphi_{x_{1}} u^{n+1}+\varphi u_{x_{1}}^{n+1} \\
\vdots & & \vdots \\
\varphi_{x_{n}} u^{1}+\varphi u_{x_{n}}^{1} & \cdots & \varphi_{x_{n}} u^{n+1}+\varphi u_{x_{n}}^{n+1}
\end{array}\right] .
$$

Here the vectors $T_{k}(u(x))$ and $\varphi_{x_{j}}(x) u(x)$ are proportional for a.e. $x$ and each $1 \leq j \leq n$, so by elementary properties of the determinant the result follows.

\section{Proof of the Theorem 1.3}

We start by proving the local boundedness of a solution, which as mentioned in the Introduction is the main content of Theorem 1.3.

Theorem 3.1. Under the assumptions of Theorem 1.3 any weak solution is locally bounded.

Proof. We can split the proof in three principal steps.

Step 1. Let $B_{r}\left(x_{0}\right)$ be such that $B_{2 r}\left(x_{0}\right) \Subset \Omega$ and

$$
\int_{B_{2 r}\left(x_{0}\right)}|\nabla u|^{n} \mathrm{~d} x \leq \varepsilon_{0},
$$

where $\varepsilon_{0}>0$ will be chosen in the course of the proof. Without loss of generality we can assume $x_{0}=0$ and denote $B_{r}\left(x_{0}\right)$ and $B_{2 r}\left(x_{0}\right)$ simply by $B_{r}$ and $B_{2 r}$, respectively. Let us denote by $\bar{u}$ the extension of $u$ from $B_{2 r}$, given in Lemma 2.4.

Let $\varphi \in \mathrm{W}_{0}^{1, n}\left(B_{2 r}\right) \cap \mathrm{L}^{\infty}\left(B_{2 r}\right)$, extended by 0 outside $B_{2 r}$ and still denoted by $\varphi, \varphi \geq 0$, such that $\int_{B_{2 r}}|\nabla u|^{n-1}|\nabla \varphi||u| \mathrm{d} x<\infty$ and $\int_{B_{2 r}}|\nabla \varphi|^{n}|u|^{n} \mathrm{~d} x<+\infty$. We observe that $T_{k}(u) \varphi^{n} \in$ $\mathrm{W}_{0}^{1, n}\left(\Omega, \mathbb{R}^{n+1}\right) \cap \mathrm{L}^{\infty}\left(\Omega, \mathbb{R}^{n+1}\right)$ and so we can use it as test function in the equation satisfied by $u$ and write the integrals on the whole $\mathbb{R}^{n}$ using the extensions of $u$ and $\varphi$ outside $B_{2 r}$, respectively. We get

$$
\begin{aligned}
\int_{\mathbb{R}^{n}}|\nabla \bar{u}|^{n-2} \nabla \bar{u} \cdot \nabla\left(T_{k}(\bar{u})\right) \varphi^{n} \mathrm{~d} x & +n \int_{\mathbb{R}^{n}}|\nabla \bar{u}|^{n-2} \nabla \bar{u} \cdot\left(T_{k}(\bar{u}) \otimes \nabla \varphi\right) \varphi^{n-1} \mathrm{~d} x \\
& =n^{\frac{n}{2}} \int_{\mathbb{R}^{n}} H(\bar{u}) \varphi^{n} T_{k}(\bar{u}) \cdot J_{\bar{u}} \mathrm{~d} x \\
& =n^{\frac{n}{2}} \int_{\mathbb{R}^{n}} H(\bar{u}) T_{k}(\bar{u}) \cdot J_{\varphi \bar{u}} \mathrm{~d} x,
\end{aligned}
$$


where the last equality follows by Lemma 2.5. Using (2.2), (2.1) and Lemma 2.3, we can estimate the last term as

$$
\begin{aligned}
\int_{\mathbb{R}^{n}} H(\bar{u}) T_{k}(\bar{u}) \cdot J_{\varphi \bar{u}} \mathrm{~d} x & \leq C\left\|H(\bar{u}) T_{k}(\bar{u})\right\|_{\mathrm{BMO}}\|\nabla(\varphi \bar{u})\|_{\mathrm{L}^{n}\left(\mathbb{R}^{n}\right)}^{n} \\
& \leq C \omega\left(\int_{\mathbb{R}^{n}}|\nabla \bar{u}|^{n}\right)^{\frac{1}{n}}\|\nabla(\varphi \bar{u})\|_{\mathrm{L}^{n}\left(\mathbb{R}^{n}\right)}^{n} \\
& \leq C \omega\left(\int_{B_{2 r}}|\nabla u|^{n} \mathrm{~d} x\right)^{\frac{1}{n}}\|\nabla(\varphi u)\|_{\mathrm{L}^{n}\left(B_{2 r}\right)}^{n} \\
& \leq C \omega\left(\varepsilon_{0}\right)\|\nabla(\varphi u)\|_{\mathrm{L}^{n}\left(B_{2 r}\right)}^{n} .
\end{aligned}
$$

Then, thanks to the hypotheses on $\varphi$, we can pass to the limit as $k \rightarrow \infty$ to get

$$
\int_{B_{2 r}}|\nabla u|^{n} \varphi^{n} \mathrm{~d} x+n \int_{B_{2 r}}|\nabla u|^{n-2} \nabla u \cdot(u \otimes \nabla \varphi) \varphi^{n-1} \mathrm{~d} x \leq C \omega\left(\varepsilon_{0}\right) \int_{B_{2 r}}|\nabla(\varphi u)|^{n} \mathrm{~d} x .
$$

Step 2. Now we can choose $\varphi=T_{k}(|u|)^{p} \eta$, where $p \geq 1$ and $\eta \in \mathrm{C}_{c}^{\infty}\left(B_{2 r}\right)$ is a cut off function between $B_{r}$ and $B_{2 r}$, in the previous estimate. Then we find

$$
\begin{aligned}
\int_{B_{2 r}}|\nabla u|^{n} T_{k}(|u|)^{p n} \eta^{n} \mathrm{~d} x & +n p \int_{\{|u| \leq k\} \cap B_{2 r}}|\nabla u|^{n-2} \nabla u \cdot(u \otimes(u \nabla u))|u|^{p n-2} \eta^{n} \mathrm{~d} x \\
& +n \int_{B_{2 r}}|\nabla u|^{n-2} \nabla u \cdot(u \otimes \nabla \eta) T_{k}(|u|)^{p n} \eta^{n-1} \mathrm{~d} x \\
\leq & C \omega\left(\varepsilon_{0}\right)\left[\int_{B_{2 r}}\left(|\nabla u|^{n} T_{k}(|u|)^{p n} \eta^{n}+|\nabla \eta|^{n} T_{k}(|u|)^{p n}|u|^{n}\right) \mathrm{d} x\right. \\
& \left.\quad+p^{n} \int_{\{|u| \leq k\} \cap B_{2 r}}|u|^{(p-1) n}|u \nabla u|^{n} \eta^{n} \mathrm{~d} x\right] \\
& \leq C \omega\left(\varepsilon_{0}\right)\left[\int_{B_{2 r}}|\nabla u|^{n} T_{k}(|u|)^{p n} \eta^{n} d x+\int_{B_{2 r}}|\nabla \eta|^{n}|u|^{p n+n} \mathrm{~d} x\right],
\end{aligned}
$$

where now the constant $C$ also depends on $p$. Observe that the second term in the left-hand side is nonnegative while the third one on the left-hand side, can be estimated by means of Young's inequality,

$$
\begin{aligned}
n \int_{B_{2 r}}|\nabla u|^{n-2} \nabla u \cdot(u \otimes \nabla \eta) T_{k}(|u|)^{p n} \eta^{n-1} \mathrm{~d} x \geq & -\frac{1}{2} \int_{B_{2 r}}|\nabla u|^{n} T_{k}(|u|)^{p n} \eta^{n} \mathrm{~d} x \\
& -c \int_{B_{2 r}}|\nabla \eta|^{n}|u|^{p n+n} \mathrm{~d} x .
\end{aligned}
$$

The first term can be absorbed in the left hand side of (3.2). Likewise with the first term in the right-hand side of (3.2), provided $\varepsilon_{0}>0$ is suitably small. At last we gain

$$
\int_{B_{r}}|\nabla u|^{n} T_{k}(|u|)^{p n} \mathrm{~d} x \leq C \int_{B_{r}}|\nabla \eta|^{n}|u|^{p n+n} \mathrm{~d} x,
$$

so that in particular, after letting $k$ tend to infinity, $|\nabla u|^{n}|u|^{p n} \in \mathrm{L}^{1}\left(B_{r}\right)$.

Step 3. The main ingredient is the construction of a suitable test function, and our argument is modelled on $[3,8]$. It is well-known that, working with precise representatives, we have for the pointwise restrictions, $\left.u\right|_{\partial B_{r}} \in \mathrm{W}^{1, n}\left(\partial B_{r}, \mathbb{R}^{n+1}\right)$ hold for almost all radii $r \in\left(0, \operatorname{dist}\left(x_{0}, \partial \Omega\right)\right)$. By Morrey's embedding we then in particular have for such radii that $\left.u\right|_{\partial B_{r}}$ is bounded. We fix such a radius $r$ which is simultaneously so small that also $\int_{B_{r}}|\nabla u|^{n} \mathrm{~d} x<\varepsilon_{0}$. Fix $R_{0} \geq\|u\|_{\mathrm{L}^{\infty}\left(\partial B_{r}\right)}$. Let $R \geq R_{0}$ and $\alpha \in(0,1]$. Choose a map $\Phi \in \mathrm{C}^{1}$ such that $\Phi(s)=0$ for $s \leq R, \Phi(s)=1$ for $s \geq(1+\alpha) R$, $\Phi^{\prime} \geq 0$ and $\Phi(s)+s \Phi^{\prime}(s) \leq 2 / \alpha$. We get $\Phi(|u|) \in \mathrm{W}_{0}^{1, n}\left(B_{r}\right) \cap \mathrm{L}^{\infty}\left(B_{r}\right)$ and may extend this map to all of $\mathbb{R}^{n} \backslash B_{r}$ by 0 . 
Let us note that $\Phi(|u|)$ satisfies the required conditions for the function $\varphi$ in Step 1, therefore arguing as in the proof of Step 1, the use of $\Phi(|u|)^{n+1} u$ to test the equation can be justified and we hereby estimate using also (2.1) and (2.2):

$$
\begin{aligned}
\int_{B_{r}}|\nabla u|^{n} \Phi(|u|)^{n+1} \mathrm{~d} x & +(n+1) \int_{B_{r}}|\nabla u|^{n-2} \nabla u \cdot\left(u \otimes\left(\frac{u \nabla u}{|u|}\right)\right) \Phi^{\prime}(|u|) \Phi(|u|)^{n} \mathrm{~d} x \\
& \leq C\|H(\bar{u}) \bar{u} \Phi(|u|)\|_{\mathrm{BMO}}\|\nabla(\Phi(|u|) \bar{u})\|_{\mathrm{L}^{n}\left(\mathbb{R}^{n}\right)}^{n} .
\end{aligned}
$$

Observe that the second term on the left-hand side is nonnegative. To estimate the BMO norm we proceed using Poincaré's inequality:

$$
\|H(\bar{u}) \bar{u} \Phi(|u|)\|_{\text {BMO }} \leq C\left(\int_{B_{r} \cap\{|u|>R\}}|\nabla(H(u) u \Phi(|u|))|^{n} \mathrm{~d} x\right)^{\frac{1}{n}} .
$$

Here $H(u) u \Phi(|u|)$ is in $\mathrm{W}_{0}^{1, n}\left(B_{r}, \mathbb{R}^{n+1}\right)$ by virtue of Step 2 since

$$
\begin{aligned}
|\nabla(H(u) u \Phi(|u|))|^{n} \leq & c\left|H^{\prime}(u)\right|^{n}|\nabla u|^{n} \Phi(|u|)^{n}|u|^{n}+c\left|\Phi^{\prime}(|u|) u\right|^{n}|\nabla u|^{n}|H(u)|^{n}+ \\
& +c|H(u)|^{n} \Phi(|u|)^{n}|\nabla u|^{n} \leq \frac{C}{\alpha^{n}}|\nabla u|^{n}\left(|u|^{n+n q}+1\right) \chi_{B_{r} \cap\{|u|>R\} .}
\end{aligned}
$$

Thus we get

$$
\begin{aligned}
\|H(\bar{u}) \bar{u} \Phi(|u|)\|_{\mathrm{BMO}} & \leq \frac{C}{\alpha}\left(\int_{B_{r} \cap\{|u|>R\}}|\nabla u|^{n-1}|\nabla u|\left(|u|^{n+n q}+1\right) \mathrm{d} x\right)^{\frac{1}{n}} \\
& \leq \frac{C}{\alpha}\left(\int_{B_{r} \cap\{|u|>R\}}|\nabla u|^{n} \mathrm{~d} x\right)^{\frac{n-1}{n^{2}}}\left(\int_{B_{r}}|\nabla u|^{n}\left(1+|u|^{n^{2}+n^{2} q}\right) \mathrm{d} x\right)^{\frac{1}{n^{2}}} .
\end{aligned}
$$

Finally, since

we obtain

$$
\|\nabla(\Phi(|u|) \bar{u})\|_{L^{n}\left(\mathbb{R}^{n}\right)}^{n} \leq \frac{C}{\alpha^{n}} \int_{B_{r} \cap\{|u|>R\}}|\nabla u|^{n} \mathrm{~d} x
$$

$$
\int_{B_{r} \cap\{|u|>(1+\alpha) R\}}|\nabla u|^{n} \mathrm{~d} x \leq \frac{C}{\alpha^{n+1}}\left(\int_{B_{r} \cap\{|u|>R\}}|\nabla u|^{n} \mathrm{~d} x\right)^{1+\frac{n-1}{n^{2}}}
$$

where we used the fact that $|\nabla u|^{n}|u|^{n^{2}+n^{2} q} \in \mathrm{L}^{1}\left(B_{r}\right)$ by Step 2 (and we incorporated this integral into the constant). Now if we define

$$
\Lambda(R):=\int_{\{|u|>R\}}|\nabla u|^{n} \mathrm{~d} x,
$$

we obtain

$$
\Lambda((1+\alpha) R) \leq \frac{C}{\alpha^{n+1}} \Lambda(R)^{1+\frac{n-1}{n^{2}}} .
$$

Choosing $\alpha_{k}=2^{-k}$ and defining by recurrence a bounded increasing sequence $R_{k+1}=R_{k}\left(1+2^{-k}\right)$, we get

$$
\Lambda\left(R_{k+1}\right) \leq C A^{k} \Lambda\left(R_{k}\right)^{1+\beta},
$$

where $A=2^{n+1}$ and $\beta=\frac{n-1}{n^{2}}$. If $\Lambda\left(R_{0}\right) \leq C^{-\frac{1}{\beta}} A^{-\frac{1}{\beta^{2}}}$, then it follows by induction that $\Lambda\left(R_{k}\right) \leq$ $A^{-\frac{k}{\beta}} \Lambda\left(R_{0}\right)$ holds for all $k$ (see for instance [14, Lemma 7.1]). Recall that $\int_{B_{r}}|\nabla u|^{n} \mathrm{~d} x<\varepsilon_{0}$, so obviously $\Lambda\left(R_{0}\right)<\varepsilon_{0}$ too. Hence if we choose $\varepsilon_{0}$ suitably small, then we have shown that

$$
\Lambda\left(R_{\infty}\right) \leq \lim _{k} \Lambda\left(R_{k}\right)=0 \text { and therefore }|u(x)| \leq R_{\infty} \text { a.e. in } B_{r},
$$

where $R_{\infty}=\lim _{k \rightarrow+\infty} R_{k}$. 
Proof of Proposition 1.1. We will first prove a Caccioppoli inequality and fix a ball $B_{0} \Subset \Omega$ so small that

$$
\int_{B_{0}}|\nabla u|^{n} \mathrm{~d} x \leq \varepsilon_{0}
$$

holds for an $\varepsilon_{0}>0$ that will be chosen in the course of the proof. As before, let $B_{r}=B_{r}\left(x_{0}\right)$ be such that $B_{2 r}=B_{2 r}\left(x_{0}\right) \Subset B$. By our assumption $\bar{R}:=\|u\|_{\mathrm{L}^{\infty}\left(B_{0}\right)}$ is finite. Denote by $\bar{u}$ the extension of $u$ from $B_{2 r}$ given by Lemma 2.4 .

Let $\eta$ be a cut-off function between $B_{r}$ and $B_{2 r}$, choose the function $\eta^{n}\left(u-u_{2 r}\right)$, where $u_{2 r}=u_{B_{2 r}}$, as test in (1.1) (see also Remark 1.2) and write the integrals in the right-hand side of the equation on the whole of $\mathbb{R}^{n}$. We gain:

$$
\int_{B_{2 r}}|\nabla u|^{n-2} \nabla u \cdot \nabla\left(\eta^{n}\left(u-u_{2 r}\right)\right) \mathrm{d} x=n^{\frac{n}{2}} \int_{\mathbb{R}^{n}} H(\bar{u}) \eta^{n}\left(\bar{u}-u_{2 r}\right) \cdot J_{\bar{u}} \mathrm{~d} x=: \mathcal{I} .
$$

Here the left-hand side is

$\int_{B_{2 r}}|\nabla u|^{n-2} \nabla u \cdot \nabla\left(\eta^{n}\left(u-u_{2 r}\right)\right) \mathrm{d} x=\int_{B_{2 r}}|\nabla u|^{n} \eta^{n} \mathrm{~d} x+n \int_{B_{2 r}}|\nabla u|^{n-2} \nabla u \cdot\left(\eta^{n-1}\left(u-u_{2 r}\right) \otimes \nabla \eta\right) \mathrm{d} x$, and the second term on the right-hand side can be estimated by means of Young's inequality,

$n \int_{B_{2 r}}|\nabla u|^{n-2} \nabla u \cdot \eta^{n-1}\left(u-u_{2 r}\right) \otimes \nabla \eta \mathrm{d} x \geq-\frac{1}{2} \int_{B_{2 r}}|\nabla u|^{n} \eta^{n} \mathrm{~d} x-C \int_{B_{2 r}}|\nabla \eta|^{n}\left|u-u_{2 r}\right|^{n} \mathrm{~d} x$.

Thus returning to (3.4) we get

$$
\int_{B_{2 r}}|\nabla u|^{n} \eta^{n} \mathrm{~d} x \leq C \int_{B_{2 r}}|\nabla \eta|^{n}\left|u-u_{2 r}\right|^{n} \mathrm{~d} x+2 \mathcal{I} .
$$

Let us now estimate $\mathcal{I}$. We first observe

$$
\mathcal{I}=n^{\frac{n}{2}} \int_{\mathbb{R}^{n}} H(\bar{u}) \eta^{n}\left(\bar{u}-u_{2 r}\right) \cdot J_{\bar{u}-u_{2 r}} \mathrm{~d} x=n^{\frac{n}{2}} \int_{\mathbb{R}^{n}} H(\bar{u})\left(\bar{u}-u_{2 r}\right) \cdot J_{\left(\bar{u}-u_{2 r}\right) \eta} \mathrm{d} x,
$$

by Lemma 2.5. We aim to use the bounds (2.1), (2.2) and must argue that the first factor is in BMO. To that end we note that $y \mapsto H(y)\left(y-u_{2 r}\right)$ is continuous, and so in particular uniformly continuous for $|y| \leq 2 \bar{R}$. We can therefore find a modulus of continuity $\omega=\omega_{\bar{R}}:[0, \infty) \rightarrow[0, \infty)$ (an increasing, continuous and concave functions with $\omega(0)=0$ ) such that

$$
\left|H(y)\left(y-u_{2 r}\right)-H\left(y^{\prime}\right)\left(y^{\prime}-u_{2 r}\right)\right| \leq \omega\left(\left|y-y^{\prime}\right|\right)
$$

for all $y, y^{\prime}$ with $|y|,\left|y^{\prime}\right| \leq 2 \bar{R}$. Observe that $\omega$ only depends on the $\mathrm{L}^{\infty}$ bound for $u$ on the fixed ball $B_{0}$ and that $|\bar{u}| \leq 2 \bar{R}$ a.e. in $\mathbb{R}^{n}$ by our assumptions and Lemma 2.4. Now for any ball $B \subset \mathbb{R}^{n}$ we estimate as in Lemma 2.3, using also the integral bound from Lemma 2.4 and (3.1), to get

$$
f_{B}\left|H(\bar{u})\left(\bar{u}-u_{2 r}\right)-\left(H(\bar{u})\left(\bar{u}-u_{2 r}\right)\right)_{B}\right| \mathrm{d} x \leq c \omega\left(\varepsilon_{0}\right)
$$

for some dimensional constant $c=c(n)$. Consequently, $\left\|H(\bar{u})\left(\bar{u}-u_{2 r}\right)\right\|_{\mathrm{BMO}} \leq c \omega\left(\varepsilon_{0}\right)$ and we may continue with

$$
\begin{aligned}
\mathcal{I} & \leq C\left\|J_{\left(\bar{u}-u_{2 r}\right) \eta}\right\|_{\mathcal{H}^{1}}\left\|H(\bar{u})\left(\bar{u}-u_{2 r}\right)\right\|_{\mathrm{BMO}} \\
& \leq C \int_{B_{2 r}}\left|\nabla\left(\left(u-u_{2 r}\right) \eta\right)\right|^{n} \mathrm{~d} x\left(c \omega\left(\varepsilon_{0}\right)\right)^{\frac{1}{n}} \\
& \leq c \omega\left(\varepsilon_{0}\right)^{\frac{1}{n}} \int_{B_{2 r}}\left(|\nabla u|^{n} \eta^{n}+|\nabla \eta|^{n}\left|u-u_{2 r}\right|^{n}\right) \mathrm{d} x .
\end{aligned}
$$

If $\varepsilon_{0}$ is suitable small (depending on the fixed ball $B_{0}$ and the data) the first term can be absorbed in the left-hand side of (3.5). Summarising we have therefore established the following Caccioppoli inequality:

$$
\int_{B_{r}}|\nabla u|^{n} \mathrm{~d} x \leq \frac{C}{r^{n}} \int_{B_{2 r}}\left|u-u_{B_{2 r}}\right|^{n} \mathrm{~d} x
$$


where the constant $C=C\left(B_{0}\right)$ and $B_{r}=B_{r}\left(x_{0}\right)$ is any ball with $B_{2 r}\left(x_{0}\right) \subset B_{0}$. Now using Poincaré-Sobolev's inequality and Gehring's Lemma (see for instance [17]) we find in a routine manner that $\nabla u \in \mathrm{L}_{\text {loc }}^{n+\delta}\left(\Omega, \mathbb{R}^{(n+1) \times n}\right)$ for some $\delta>0$. By Morrey's embedding theorem this implies that $u$ is $\mathrm{C}_{\text {loc }}^{0, \beta}\left(\Omega, \mathbb{R}^{n+1}\right)$ for some $\beta<1$.

It is well-known that the asserted $\mathrm{C}^{1, \alpha}$ regularity follows from this when $H$ is locally Lipschitz. A streamlined approach can be obtained following [11, Lemma 5] to get that $u$ is $\mathrm{C}_{\text {loc }}^{0, s}\left(\Omega, \mathbb{R}^{n+1}\right)$ for each $s<1$, and then finally [11, Lemma 6] to conclude that $u$ is $\mathrm{C}_{\text {loc }}^{1, \alpha}\left(\Omega, \mathbb{R}^{n+1}\right)$ for some $\alpha \in(0,1)$.

The proof of Theorem 1.3 follows by combination of Theorem 3.1 and Proposition 1.1.

\section{EXAMPLE}

The condition of Heinz and Wang on the $\mathrm{C}^{1}$ function $H: \mathbb{R}^{n+1} \rightarrow \mathbb{R}$ is

$$
\sup _{y \in \mathbb{R}^{n+1}}\left(|H(y)|+\left|H^{\prime}(y)\right|(1+|y|)\right)<\infty .
$$

We can rewrite this and split it into two assumptions as

$$
\left\{\begin{array}{l}
H \text { Lipschitz } \\
y \mapsto H(y)(|y|+1) \text { Lipschitz continuous. }
\end{array}\right.
$$

Here we give an example of a $\mathrm{C}^{1}$ function $H$ that does not satisfy the assumptions of Heinz and Wang but is such that our Theorem 1.3 in particular applies to it:

$$
\left\{\begin{array}{l}
H \text { Lipschitz } \\
y \mapsto H(y)(|y|+1) \text { uniformly continuous. }
\end{array}\right.
$$

Example: Let $\theta: \mathbb{R} \rightarrow \mathbb{R}$ be even and

$$
\theta(t):= \begin{cases}1 & 0 \leq t \leq 1 \\ t^{2}(t-2)^{2} & 1 \leq t \leq 2 \\ 0 & t \geq 2\end{cases}
$$

Then $\theta \in C^{1}, 0 \leq \theta(t) \leq \mathbf{1}_{(-2,2)}(t)$, sup $\left|\theta^{\prime}(t)\right|=\left|\theta^{\prime}\left( \pm t_{0}\right)\right|=\theta_{0}$, where $t_{0}=(3+\sqrt{3}) / 3 \in(1,2)$ and $\theta_{0}=\frac{8}{9} \sqrt{3}$. Fix an increasing function $\omega:(0,1] \rightarrow(0,1]$ with $\omega\left(0^{+}\right)=0$. Note that the intervals $\left[2^{j}-2^{1-j}, 2^{j}+2^{1-j}\right]$ are pairwise disjoint and that none of them contains 0 when $j \in \mathbb{N}$. The function

$$
H(y):=\sum_{j=1}^{\infty} \omega\left(2^{-j}\right) \frac{\theta\left(2^{j}|y|-2^{2 j}\right)}{1+|y|}, \quad y \in \mathbb{R}^{n+1},
$$

is therefore easily seen to be $\mathrm{C}^{1}$ and $0 \leq H(y)(1+|y|) \leq 1$ for all $y \in \mathbb{R}^{n+1}$.

Now if $\omega$ is superlinear at 0 , then $F(y):=H(y)(1+|y|)$ cannot be Lipschitz since for $y_{\ell} \in \mathbb{R}^{n+1}$ with $\left|y_{\ell}\right|=2^{\ell}+\frac{3+\sqrt{3}}{3} 2^{-\ell}$ we have that $\left|F^{\prime}\left(y_{\ell}\right)\right|=\theta_{0} \omega\left(\frac{1}{2^{\ell}}\right) 2^{\ell}$ which is unbounded for $\ell \in \mathbb{N}$. However, it is not difficult to check that $F$ is uniformly continuous: let $\varepsilon>0$, and take $s \in N$ with $\omega\left(2^{-s}\right) \leq \varepsilon$. Since $F$ is Lipschitz on $\left[-2^{s}, 2^{s}\right]$ we can find $\delta_{1}>0$ so

$$
\left|F\left(y_{1}\right)-F\left(y_{2}\right)\right| \leq \varepsilon \quad \forall y_{1}, y_{2} \in\left[-2^{s}, 2^{s}\right], \quad\left|y_{1}-y_{2}\right| \leq \delta_{1} .
$$

Next, for $y_{2} \in \mathbb{R}^{n+1}$ with $\left|y_{2}\right|>2^{s}$ we note that for any $y_{1} \in \mathbb{R}^{n+1}$ with $\left|y_{1}-y_{2}\right|<2^{-s}$ we have that $\left|F\left(y_{1}\right)-F\left(y_{2}\right)\right| \leq C \omega\left(2^{-s}\right) \leq C \varepsilon$. Taking $\delta=\min \left(\delta_{1}, 2^{-s}\right)$ we fend off $\varepsilon$ and conclude that $F$ is uniformly continuous on $\mathbb{R}^{n+1}$. 


\section{REFERENCES}

[1] L. Beck and J. Frehse, Regular and irregular solutions for a class of elliptic systems in the critical dimension, NoDEA Nonlinear Differential Equations Appl. 20 (2013), no. 3, 943-976.

[2] F. Bethuel, Un résultat de regularité pour les solutions de l'équation des surfaces à courboure moyenne prescrite, C.R. Acad. Sci. Paris Sér. I Math. 314 (1992), 1003-1007.

[3] F. Bethuel and J.M. Ghidaglia, Improved regularity of solutions to elliptic equations involving jacobians and applications, J. Math. Pure Appl. 72 (1993), 441-474.

[4] F. Bethuel, P. Caldiroli and M. Guida, Parametric surfaces with prescribed mean curvature. Turin Fortnight Lectures on Nonlinear Analysis (2001). Rend. Sem. Mat. Univ. Politec. Torino 60 (2002), no. 4, 175-231.

[5] J. Bourgain and H. Brezis, New estimates for elliptic equations and Hodge type systems, J. Eur. Math. Soc. 9 (2007), 277-315.

[6] H. Brezis and J.-M. Coron, Multiple solutions of $H$-systems and Rellich's conjecture, Comm. Pure Appl. Math. 37 (1984), 149-187.

[7] H. Brezis and H.-M. Nguyen, The Jacobian determinant revisited, Invent. Math. 185 (2011), 17-54.

[8] P. Caldiroli and R. Musina, Weak limit and blowup of approximate solutions to H -systems, J. Funct. Anal. 249 (2007),

[9] R. Coifman, P.L. Lions, Y. Meyer, S. Semmes, Compensated compactness and Hardy spaces, J. Math. Pures Appl. 72 (1993), 247-286.

[10] F. Duzaar and J.F. Grotowski, Existence and regularity for higher-dimensional H-systems, Duke Math. J. 101 (2000), no. 3 , 459-485.

[11] F. Duzaar and G. Mingione, The p-harmonic approximation and the regularity of p-harmonic maps, Calc. Var. Partial Differential Equations 20 (2004), no. 3, 235-256.

[12] C. Fefferman, Characterizations of bounded mean oscillation, Bull. Amer. Math. Soc. 77 (1971), 587-588.

[13] M. Giaquinta and S. Hildebrandt, A priori estimates for harmonic mappings, J. für Mathematik 336 (1982), 124-164.

[14] E. Giusti, Direct methods in the calculus of variations, World Scientific Publishing Co., Inc., River Edge, NJ, 2003. viii+403 pp.

[15] M. Grüter, Regularity of weak H -surfaces, J. Reine Angew Math. 329 (1981), 1-15.

[16] E. Heinz, Ein Regularitätssatz für schwacher lösungen nichtlinearer elliptischer systeme, Nachr. Akad. Wiss. Göttingen Math. Phys. KL II (1) (1975), 1-13.

[17] T. Iwaniec and G. Martin, Geometric function theory and non-linear analysis, Oxford Mathematical Monographs, The Clarendon Press, Oxford University Press, New York, 2001. xvi+552.

[18] C. Leone, M. Misawa and A. Verde, A global existence result for the heat flow of higher dimensional H-systems, J. Math. Pures Appl. (9) 97 (2012), no. 3, 282-294.

[19] V.G. Maz'ya, Sobolev spaces with applications to elliptic partial differential equations. Second, revised and augmented edition, Grundlehren der Mathematischen Wissenschaften [Fundamental Principles of Mathematical Sciences], 342 Springer, Heidelberg, 2011. xxviii+866 pp.

[20] L. Mou and P. Yang, Multiple solutions and regularity of $H$-systems, Indiana Univ. Math. J. 45 (4) (1996), 1193-1222.

[21] T. Rivière, Conservation laws for conformally invariant variational problems, Invent. Math. 168 (2007), no. 1, 1-22.

[22] A. Schikorra and P. Strzelecki, Invitation to H-systems in higher dimensions: known results, new facts, and related open problems, EMS Surv. Math. Sci. 4 (2017), no. 1, 21-42.

[23] E.M. Stein, Harmonic analysis: real-variable methods, orthogonality, and oscillatory integrals. With the assistance of Timothy S. Murphy, Princeton Mathematical Series, 43, Monographs in Harmonic Analysis, III, Princeton University Press, Princeton, NJ, 1993, xiv+695 pp.

[24] P. Strzelecki, A new proof of regularity of weak solutions of the $H$-surface equation, Calc. Var. Partial Diff. Eq. 16 (2003), no. 3, 227-242.

[25] M. Struwe, Variational methods. Applications to nonlinear partial differential equations and Hamiltonian systems. Fourth edition. Ergebnisse der Mathematik und ihrer Grenzgebiete, 3. Folge. A Series of Modern Surveys in Mathematics [Results in Mathematics and Related Areas. 3rd Series. A Series of Modern Surveys in Mathematics], 34, Springer-Verlag, Berlin, 2008. $x x+302 \mathrm{pp}$.

[26] F. Tomi, Bemerkungen zum regularitäts problem der Gleichung vorgeschriebener mittlerer Krümmung, Math. Z. 132 (1973), 323-326.

[27] C. Wang, Regularity of high-dimensional $H$-systems, Nonlinear Anal. 38 (1999), 675-686.

[28] H.C. Wente, An existence theorem for surface of constant mean curvature, J. Math. Anal. Appl. 26 (1969), 318-344.

[29] W.P. Ziemer, Weakly Differentiable Functions. Sobolev Spaces and Functions of Bounded Variation, Graduate Texts in Math. 120, Springer-Verlag, New York, 1989. 